\title{
Additional realizations of single-element-controlled oscillators using single ICCII-
}

\author{
Abhirup Lahiri, Member, IACSIT
}

\begin{abstract}
This paper presents additional realizations of single-element-controlled oscillators (SECOs) using a single negative-type inverting second-generation current conveyor (ICCII-). The circuits have been derived from a single ICCIIbased topology previously proposed by Toker et al. Both single-resistance-controlled oscillators (SRCOs) and SECOs have been obtained. The circuits use one ICCII- and five/six external passive components, hence reduced number of components are employed. The circuits also provide explicit-current-output (ECO) and enjoy low active and passive sensitivities. PSPICE experimental results have been included to verify the theoretical results.
\end{abstract}

Index Terms - Active RC circuits, Single-element-controlled oscillators (SECOs), inverting second-generation current conveyor (ICCII)

\section{INTRODUCTION}

Sinusoidal oscillators find numerous applications in communication, control systems, signal processing, instrumentation and measurement systems and the applications and advantages in realizing RC sinusoidal oscillators using second generation current conveyors (CCIIs) have received considerable attention [1]-[7] in the past. In 1999, Awad and Soliman introduced a new active building block (ABB), namely the inverting second generation current conveyor (ICCII) and which is an additional block to the current conveyor family [8]. This $\mathrm{ABB}$ besides possessing the advantages of its predecessor (CCII) had additional advantageous features of obtaining current-mode circuits from their voltage-mode counterparts by using the ad joint network theorem [9] as explained in [8] Several circuit solutions using ICCII have been reported in the literature [10]-[12]. The aim of this brief is to present new single-resistance-controlled oscillators (SRCOs) and single-element-controlled oscillators (SECOs) using this versatile ABB. SRCOs provide independent control of condition of oscillation (CO) and frequency of oscillation (FO) by means of different resistors and SECOs provide single-element-control of oscillation frequency by either a resistor or a capacitor. The proposed oscillators are additions to the previously reported circuits in [13], which are categorized according to the number of ABBs used. But

Manuscript received, $21^{\text {st }}$ March, 2009.

Abhirup Lahiri, is currently with the Division of Electronics and Communications, Netaji Subhas Institute of Technology (NSIT), University of Delhi, New Delhi, India.. most of the SRCOs or SECOs presented in [13] make use of multiple ABBs. One single ICCII based SRCO is also provided in [10] (see Fig. 3, topology 2 of [13]), but a detailed analysis of the proposed topology indicates that more SRCOs/SECOs could be derived from the same structure and hence the motivation is to come up with more oscillator circuits having single-element-control over condition of oscillation and frequency of oscillation, from the existing single negative type ICCII (ICCII-) based topology in [13]. Total of six oscillator circuits are reported in this brief, which include both SRCOs and SECOs. The oscillator circuits use reduced number (five/six) of external passive components (only resistors and capacitors). The circuits are also capable of providing explicit-current-output (ECO) by use of a dual-output ICCII and they could be used as current-mode oscillators in various current-mode signal processing applications. Numerous applications of the proposed circuits have been provided and the non-ideal analysis and sensitivity study have been carried out. PSPICE simulations have been included which verify the workability of the circuits.

\section{INVERTING SECOND-GENERATION CURRENT CONVEYOR (ICCII)}

The ICCII was first introduced in [8] and in this brief its variant, namely the dual-output negative type ICCII i.e. DOICCII- is used which is characterized by the following

$$
\left(\begin{array}{c}
I_{y} \\
V_{x} \\
I_{z+}, I_{z-}
\end{array}\right)=\left(\begin{array}{ccc}
0 & 0 & 0 \\
-1 & 0 & 0 \\
0 & -1 & 0
\end{array}\right)\left(\begin{array}{c}
V_{y} \\
I_{x} \\
V_{z}
\end{array}\right)
$$

The circuit symbol of dual-output ICCII- is shown in Fig. 1 and the CMOS implementation could be found in [8], [12] and [13].

\section{PROPOSED CONFIGURATION}

The single ICCII- based topology is shown in Fig. 2. It is a general structure and various oscillator circuits can be derived out of it by appropriately choosing the admittances. As pointed in [13], this topology is meaningful only with ICCII but not with CCII since the admittance between $y$ and $x$ terminals of the conveyor is redundant for the latter. The possible SRCOs and SECOs have been attempted in this paper. The characteristic equation for the circuit shown in Fig. 2, in terms of the admittances can be found as 


$$
y_{1} y_{3}+y_{5}\left(y_{1}+y_{3}+2 y_{2}\right)=y_{1} y_{4}
$$

The various SRCOs and SECOs derived from this topology by choosing different combinations of passive components (the resistors and capacitors), along with the condition of oscillation (CO) and frequency of oscillation have been tabulated in Table I. From Fig. 2, it is clear that all the oscillators provide an explicit-current-output (ECO) by means of a duplicate current terminal $z_{2}$ (high output impedance terminal). This classifies the circuit as a current-mode oscillator and makes it suitable to be used in various current-mode signal processing applications [14]. As an example, these circuits could be used as sinusoidal input current-sources for current-mode active filters.

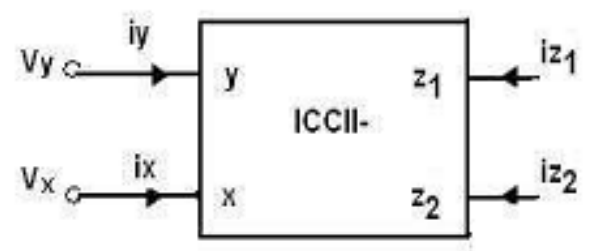

Fig. 1: Symbolic Representation of dual-output ICCII-

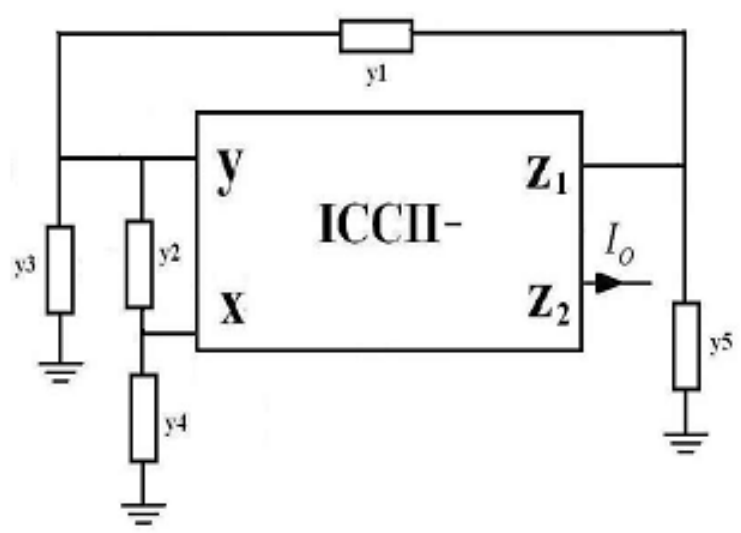

Fig. 2: Proposed single ICCII- based oscillator topology

Although CO and FO control by means of resistors is more easy and preferable, but as pointed in [15], circuits with $\mathrm{CO}$ or $\mathrm{FO}$ control by means of capacitors are not out-classed when considered from the perspective of instrumentation and measurement applications, as single-element-control of FO through a capacitor can be used as transducer oscillators in conjunction with capacitive transducers, those providing $\mathrm{CO}$ control through a capacitor can be used in some capacitance measurement schemes such as mentioned in [15]-[16] and references provided therein. The circuit number 1 (see Table 1) is a true SRCO with CO and FO controls by means of different resistors. This circuit is the same as that proposed in (Fig. 3, topology 2) [13]. For the circuit number 2, the $\mathrm{CO}$ and $\mathrm{FO}$ tuning laws indicate that it is a SECO with single-element-control of the oscillation frequency by means of $R_{2}$. This oscillator has another unique application: since the numerator of FO has a difference term, the circuit could be used as a very low frequency oscillator (VLFO) [17]. The circuit number 3 works as a SECO under the appropriate matching constraints, but it uses grounded capacitors which makes it suitable for monolithic integration [12] and [18]. Both circuit number 2 and 3 could be easily modified to create voltage-controlled oscillators (VCOs), by replacing resistor $R_{4}$ and $R_{3}$ respectively with a non-linearity cancelled MOSFET (working in triode-region) [19]-[20]. VCOs have wide applications, example in phase-locked loop (PLL), A/D convertors etc. as pointed in [15]. Additional oscillator circuits could be created from the circuits 1,2 and 3 by simple applying the RC-CR transformation, i.e. replacing capacitors by resistors and vice-versa. This would lead to three new SECOs, all of which would have FO control by means of a capacitor. Hence a total of six new oscillator circuits have been derived from a single ICCII- based configuration.

\section{NON-IDEAL ANALYSIS AND SENSITIVITY STUDY}

Considering the non-idealities of ICCII- as pointed in [8] and

[13], then $V_{x}=-\alpha V_{y}, I_{z 1}=-\beta I_{x}$ where $\alpha$ and $\beta$ differ from their ideal values of unity of voltage-tracking and current-tracking errors respectively. Taking into account the above non-ideal effects, the basic characteristic equation of the oscillator topology as in (2) gets modified to

$$
\begin{aligned}
& y_{1} y_{3}+y_{5}\left(y_{1}+y_{3}+(1+\alpha) y_{2}\right)= \\
& \alpha \beta y_{1} y_{4}+(1+\alpha)(\beta-1) y_{1} y_{2}
\end{aligned}
$$

Both the CO and FO corresponding to every circuit get modified in the non-ideal case and could be easily derived using (3), the final results of which have been provided in Table II. It is evident from Table II, that only the FO of circuit number 3 remains unaffected from the non-idealities. Also, the sensitivity study indicates that the circuits enjoy low (less than unity) $f_{o}$ active and passive sensitivities (see Table III). The non-ideal analysis and sensitivity study of the three additional SECOs created by RC-CR transformation from circuits 1,2 and 3, could be carried out in a similar manner.

\section{Simulation Results}

The working of all the proposed circuits as sinusoidal oscillators has been verified by PSPICE simulations. The circuit number 1, the SRCO employing five passive components is chosen as a design example. The simulations have been performed using a CMOS realization of ICCII- as provided in [12] and the parameters like the aspect ratio, biasing current and supply voltages were also chosen as in [12]. The circuit has been designed with the following passive component values: $C_{1}=C_{3}=\ln F$ and $R_{2}=R_{4}=1 K \Omega$, $R_{5}=2 K \Omega$. With these component values the $\mathrm{CO}$ is satisfied and theoretical frequency of oscillation is $159.1 \mathrm{KHz}$. The typical waveform of the voltage at terminal $z_{l}$ depicting the steady-state sinusoidal oscillations is shown in Fig. 3. As pointed earlier the circuit no. 1 is SRCO with FO control by means of $G_{2}$; the variation of the FO with resistor $R_{2}$ is shown in Fig. 4 and simulated values have close 
correspondence with theoretical values.

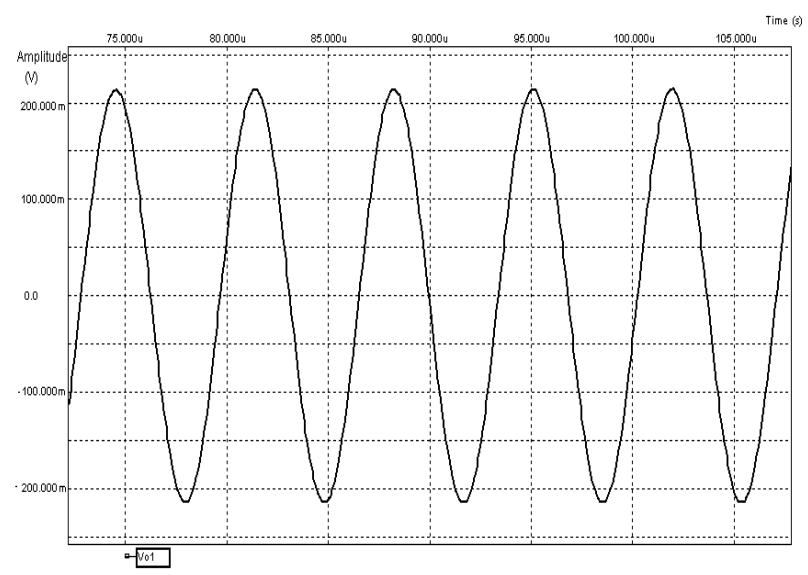

Fig.3 Steady-state time domain waveform

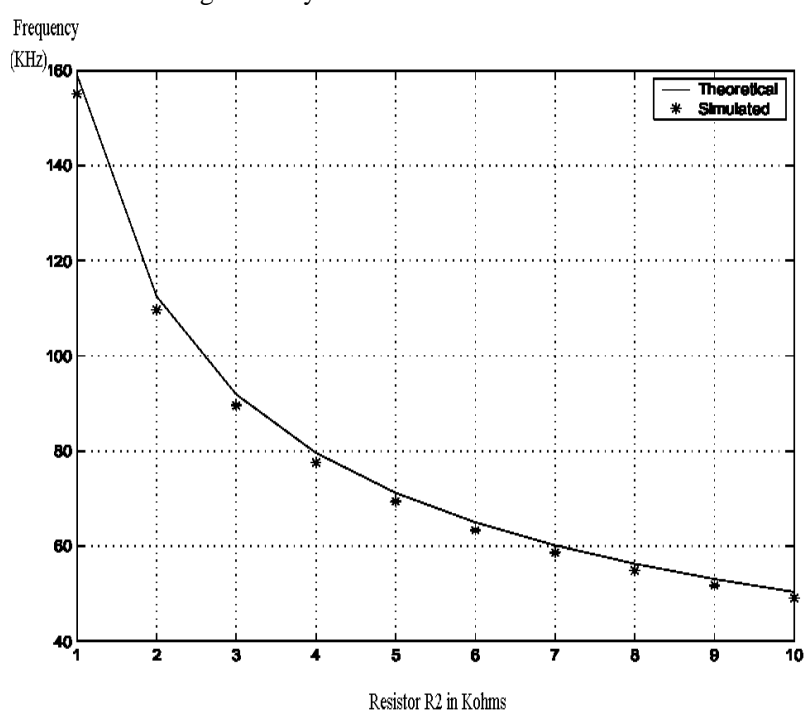

Fig. 4 Variation of FO with R2

\section{CONCLUSIONS}

New single-element-controlled oscillator circuits have been devised from a previously proposed single negative type inverting current conveyor (ICCII-) based topology. The circuits enjoy single-element-control of oscillation of frequency and are suitable to be used as variable frequency oscillators. Oscillator circuits with advantageous features to be used as very-low frequency oscillators and/or voltage-controlled oscillators have also been created. All the circuits provide an explicit-current-output which could be used as an input/test sinusoidal signal in many current-mode circuits, particularly in current-mode filters. The circuits also exhibit low active and passive $f_{o}$ sensitivities. PSPICE simulations have verified the workability of the circuits. The circuits add to the current repertoire of ICCII based oscillators circuits and the author is currently working on creating canonic oscillator structures using ICCII and two grounded capacitors- which shall be reported soon.

\section{ACKNOWLEDGMENT}

The author would like to thank Prof. Raj Senani, the Director of Netaji Subhas Institute of Technology (NSIT), who has always been a source of immense motivation and academic help.

\section{REFERENCES}

[1] R. Senani and V. K. Singh, "Single-element-controlled sinusoidal oscillator employing a single current conveyor IC", Electronics Letters, vol. 29, no. 14, pp. 414-415, 1992.

[2] D. R. Bhaskar and R. Senani, "New current-conveyor-based single resistance-controlled/ voltage controlled oscillator employing grounded capacitors", Electronics Letters, vol. 29, no. 7, pp. 612-614, 1993.

[3] M. T. Abuelmaatti, A. A. Al-Ghumaiz and M. H., Khan, "Novel CCII - based single element controlled oscillators employing grounded resistors and capacitors", International Journal of Electronics, vol. 78, pp. 1107-1112, 1995.

[4] A. M. Soliman, "Current-mode oscillator using single output current conveyor", Microelectronics Journal, vol. 29, pp. 907-912, 1998.

[5] M. T. Abuelma'atti, "New sinusoidal oscillators with fully uncoupled control of oscillation frequency and condition using three CCII+s", Analog Integrated Circuits and Signal Processing, vol. 24, pp. 253-261, 2000.

[6] J. W. Horng, "A sinusoidal oscillator using current controlled conveyors", International Journal of Electronics, vol. 88, pp. 659-664, 2001 .

[7] A. A. Khan, S. Bimal, K. K. Dey, and S. S. Roy, "Novel RC Sinusoidal Oscillator Using Second-Generation Current Conveyor", IEEE Transactions On Instrumentation and Measurement, vol. 54, pp. 2402-2406, 2005.

[8] I. A. Awad and A..M. Soliman, "The inverting second generation current conveyors: the missing building blocks, CMOS realizations and applications", International Journal of Electronics, vol. 84, pp. 413-432, 1999.

[9] A. Carlosena and G. Moschytz, "Nullators and norators in voltage to current mode transformations", International Journal of Circuit Theory and Applications, vol. 21, pp. 421-424, 1993.

[10] S. Minaei, E. Yuce and O. Cicekoglu, "ICCII based voltage-mode filter with single input and six outputs employing grounded capacitors", Circuits, Systems and Signal Processing, vol. 25, no. 4, pp. 559-566, 2006.

[11] E. Yuce and S. Minaei, "ICCII-Based Universal Current-Mode Analog Filter Employing Only Grounded Passive Components" Analog Integrated Circuits and Signal Processing, vol. 58, no. 2, pp. 161-169, 2009.

[12] A. M. Soliman, "New Grounded Capacitor Current Mode Band-Pass Low-Pass Filters Using Two Balanced Output ICCII", Journal of Active and Passive Electronic Devices, vol. 3, pp. 175-184, 2008

[13] A. Toker et al, "New Oscillator Topologies Using Inverting Second-Generation Current Conveyors", Turkish Journal of Electrical Engineering and Computer Sciences, vol. 10, no. 1, pp. 119-130, 2002.

[14] A. Lahiri, "New current-mode quadrature oscillators using CDTA", IEICE Electronics Express, vol. 6, no. 3, pp. 135-140, 2009.

[15] D. R. Bhaskar and R. Senani, "New CFOA-Based Single-Element-Controlled Sinusoidal Oscillators", IEEE Transactions on Instrumentation and Measurement, vol. 55, no. 6, pp. 2014-2021, 2006.

[16] W. Ahmad, "A new simple technique for capacitance measurement", IEEE Transactions on Instrumentation and Measurement, vol. 35, no. 4, pp. 640-642, 1986.

[17] A. S. Elwakil, "Systematic realization of low-frequency oscillators using composite passive-active resistors", IEEE Transactions on Instrumentation and Measurement, vol. 47, no. 2, pp. 584-586, 1998.

[18] M. Bhushan, and R.W. Newcomb, "Grounding of capacitors in integrated circuits", Electronics Letters, vol. 3, pp. 148-149, 1967.

[19] R. Senani, "Realization of linear voltage-controlled-resistance in floating form", Electronics Letters, vol. 30, no. 23, pp. 1909-1911, 1994

[20] S.S. Gupta, D.R. Bhaskar and R. Senani, "New voltage controlled oscillators using CFOAs". AEU- International Journal of Electronics and Communications, vol. 63, 209-217, 2009. 
Table I: CO and the FO comparisons of the proposed oscillators

\begin{tabular}{|c|c|c|c|c|c|c|c|c|c|}
\hline Circuit & $y_{1}$ & $y_{2}$ & $y_{3}$ & $y_{4}$ & $y_{5}$ & $\begin{array}{c}\text { CO } \\
\text { Fontrol } \\
\text { of CO }\end{array}$ & $\begin{array}{c}\text { Control } \\
\text { of FO }\end{array}$ \\
\hline 1. & $s C_{1}$ & $G_{2}$ & $s C_{3}$ & $G_{4}$ & $G_{5}$ & $G_{4}=\left(1+\frac{C_{3}}{C_{1}}\right) G_{5}$ & $\frac{1}{2 \pi} \sqrt{\frac{2 G_{2} G_{5}}{C_{1} C_{3}}}$ & $G_{4}$ \\
\hline 2. & $G_{1}$ & $s C_{2}$ & 0 & $s C_{4}+G_{4}$ & $s C_{5}+G_{5}$ & $2 C_{2} G_{5}=\left(C_{4}-C_{5}\right) G_{1}$ & $\frac{1}{2 \pi} \sqrt{\frac{G_{1}\left(G_{5}-G_{4}\right)}{2 C_{2} C_{5}}}$ & $C_{4}$ \\
\hline 3. & $G_{1}$ & 0 & $G_{3}+s C_{3}$ & $s C_{4}$ & $s C_{5}$ & $\begin{array}{c}C_{5} G_{3}+C_{5} G_{1} \\
+C_{3} G_{1}=C_{4} G_{1}\end{array}$ & $\frac{1}{2 \pi} \sqrt{\frac{G_{1} G_{3}}{C_{3} C_{5}}}$ & $\begin{array}{c}\text { If } G_{1}=G_{3}, \\
\text { then by } C_{4}\end{array}$ & \begin{tabular}{c}
$G_{1}, G_{3}$ \\
\hline
\end{tabular}
\end{tabular}

Table II: Modified CO and FO for the non-ideal case

\begin{tabular}{|c|c|c|}
\hline Circuit & $\mathrm{CO}$ & $\mathrm{FO}\left(f_{0}\right)$ \\
\hline 1. & $C_{1} G_{2}(1+\alpha)(\beta-1)+$ & $\frac{1}{2 \pi} \sqrt{\frac{(1+\alpha) G_{2} G_{5}}{C_{1} C_{3}}}$ \\
& $\alpha \beta C_{1} G_{4}=G_{5}\left(C_{3}+C_{1}\right)$ & \\
\hline 2. & $(1+\alpha) C_{2} G_{5}=\left(\alpha \beta C_{4}-C_{5}\right) G_{1}+$ & $\frac{1}{2 \pi} \sqrt{\frac{G_{1}\left(G_{5}-\alpha \beta G_{4}\right)}{(1+\alpha) C_{2} C_{5}}}$ \\
\hline 3. & $(1+\alpha)(\beta-1) C_{2} G_{1}$ & \\
\hline & $C_{5} G_{3}+C_{5} G_{1}+$ & $\frac{1}{2 \pi} \sqrt{\frac{G_{1} G_{3}}{C_{3} C_{5}}}$ \\
\hline
\end{tabular}

Table III: fo active and passive sensitivities

\begin{tabular}{|c|c|}
\hline Circuit & $f_{o}$ Sensitivities \\
\hline 1. & $\begin{array}{c}\left|S_{C_{1}, C_{3}, G_{2}, G_{5}}^{f_{o}}\right|=\frac{1}{2} \\
S_{\alpha}^{f_{o}}=\frac{\alpha}{2(1+\alpha)} \\
S_{\beta}^{f_{o}}=0\end{array}$ \\
\hline 2. & $\begin{array}{c}\left|S_{C_{2}, C_{5}, G_{1}}^{f_{o}}\right|=\frac{1}{2}, \\
S_{G_{5}}^{f_{0}}=\frac{G_{5}}{2\left(G_{5}-\alpha \beta G_{4}\right)}, \\
S_{G_{4}, \beta}^{f_{o}}=\frac{-\alpha \beta G_{4}}{2\left(G_{5}-\alpha \beta G_{4}\right)}, \\
S_{\alpha}^{f_{o}}=\frac{-\alpha \beta G_{4}}{2\left(G_{5}-\alpha \beta G_{4}\right)}-\frac{\alpha}{2(1+\alpha)}\end{array}$ \\
\hline 3. & $\begin{array}{c}\left|S_{C_{3}, C_{5}}^{f_{o}}\right|=\frac{1}{2} \\
\text { If } G_{1}=G_{3}, S_{G_{1}, G_{3}}^{f_{o}}=1, \\
S_{\alpha, \beta}^{f_{o}}=0\end{array}$ \\
\hline
\end{tabular}

Abhirup Lahiri is currently with the Digital Processing Group, Division of Electronics and Communications, Netaji Subhas Institute of Technology, University of Delhi. His research interests include mixed-mode circuit design, analog signal processing and noise analysis of circuits. He has authored and co-authored twenty international journal, conference papers and design ideas and has acted as a reviewer (by editor's invitation) for international journals. He is a member of IACSIT, IAENG and ACEEE 